\title{
Advanced lung adenocarcinoma with coexistent HER2 mutation and amplification and response to afatinib: a case report
}

\author{
Xiaona Liu, Yuqing Cao, Yuxin Li, Xiumei Duan \\ Department of Pathology, The First Hospital of Jilin University, Changchun 30021, China \\ Correspondence to: Xiumei Duan. Department of Pathology, The First Hospital of Jilin University, 71 Xinmin St, Changchun 30021, China. \\ Email: xmduan@jlu.edu.cn.
}

\begin{abstract}
Human epidermal growth factor receptor 2 (HER2) mutation and amplification are distinct molecular targets in lung cancer, but the specific targeted therapy for their coexistence is undetermined. Personalized targeted therapy is based on mutation type, with different mutations requiring different treatment. A 64-year-old Chinese woman was diagnosed with advanced lung adenocarcinoma. She was determined as having insertion mutations in exon 20 of the HER2 gene (c.2326G > TTGT) by the amplification refractory mutation system (ARMS) and HER2 gene amplification (HER2/CEP17 ratio 2.6) by fluorescence in situ hybridization (FISH). Thereafter, she was treated with afatinib as first-line therapy, to which she responded. After 2 months, the tumor lesion decreased in size. Computed tomography (CT) follow-up showed stable lung lesions, although she later developed multiple brain metastases and subsequently died of brain failure. Lung adenocarcinoma with coexistent HER2 mutation and amplification is relatively uncommon and has no reported cases on targeted therapy. This case was important because it showed effective response to afatinib and provides evidence to help clinicians identify the therapeutic regimen for such patients.
\end{abstract}

Keywords: Human epidermal growth factor receptor 2 (HER2); mutation; HER2 amplification; lung adenocarcinoma; afatinib; case report

Submitted Nov 18, 2019. Accepted for publication Jan 06, 2020.

doi: $10.21037 /$ apm.2020.02.14

View this article at: http://dx.doi.org/10.21037/apm.2020.02.14

\section{Introduction}

The human epidermal growth factor receptor 2 (HER2 or ERBB2) is a member of the epidermal growth factor receptor (EGFR) family and has tyrosine kinase activity. Unlike EGFR, HER3, and HER4, HER2 lacks the corresponding ligand and, instead, phosphorylates intracellular tyrosine residues and activates various signaling pathways by forming homodimers or heterodimers with other members of the EGFR family (1). Mutation in the HER2 kinase domain results in constitutive phosphorylation and activation of HER2 and EGFR, which induce proliferation, angiogenesis, and metastasis of nonsmall-cell lung cancer (NSCLC) cells (2). Three principal mechanisms of HER2 activation have been determined:
HER2 gene mutation, HER2 gene amplification, and HER2 protein overexpression; the latter two are most common in breast cancer and gastric cancer (3). The HER2 gene has been identified as one of the driving genes and potential therapeutic targets for lung cancer (4). Moreover, mutation and amplification of the HER2 gene accounted for $2-3 \%$ and $2-5 \%$, respectively, of lung adenocarcinoma cases (5). HER2 mutation and amplification are generally mutually exclusive in NSCLC. Li et al. (5) and Arcila et al. (6) demonstrated that HER2 gene mutations and amplification did not coexist in NSCLC. Suzuki et al. (7) reported that 25 of 44 lung cancer cases with HER2 mutation harbored HER 2 amplification, and 25 of 222 lung cancer cases with HER2 amplification harbored concurrent HER2 mutation; in total, the coexistence of HER2 mutation 

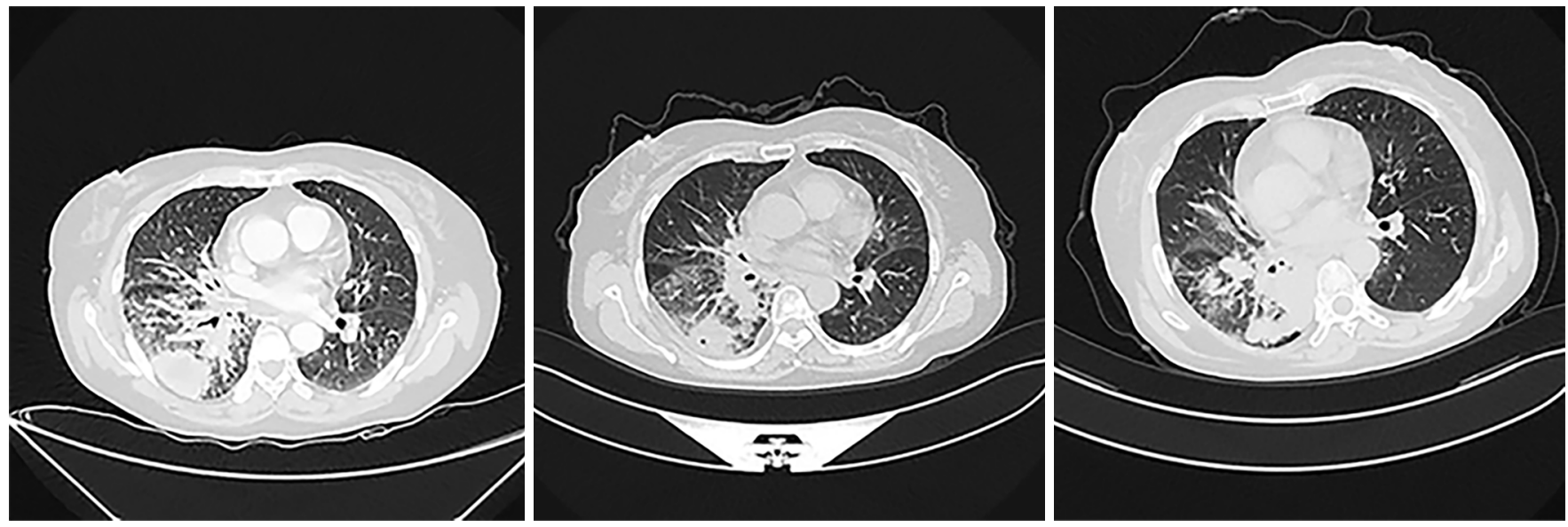

Figure 1 Lung CT images of the patient at different time points. (A) CT of chest showing the lesion in the right lower lobe when the patient arrived at our lung cancer clinic for the first time in February 2018; (B) CT of chest showing PR after 2 months of afatinib treatment; (C) CT of chest showing the lung lesion remained stable during brain metastasis. CT, computed tomography; PR, partial response.

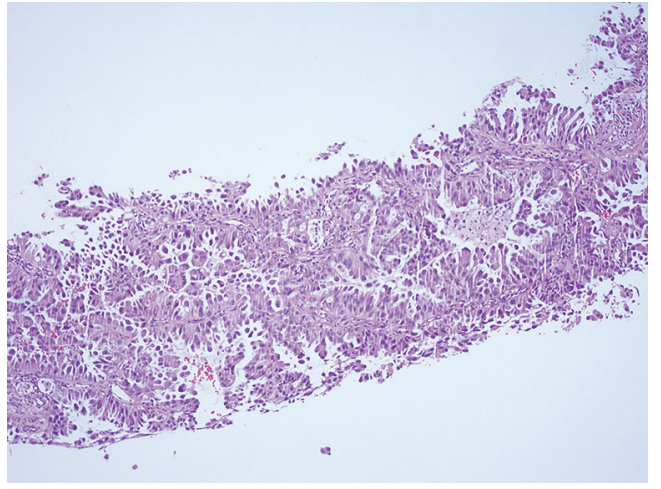

Figure 2 Pathological diagnosis by percutaneous lung puncture. Lung adenocarcinoma with an acinar and micropapillary pattern (hematoxylin-eosin staining, $\times 200$ ).

and amplification was seen in 25 of 1,170 cases, although this represented a small minority of lung cancer cases. Therefore, lung adenocarcinoma with concomitant HER2 mutation and amplification is relatively uncommon.

In this report, insertion mutation in the HER2 gene exon 20 was detected by the amplification refractory mutation system (ARMS), whereas HER2 amplification was assessed by fluorescence in situ hybridization (FISH) and was defined as a HER2 to chromosome enumeration probe 17 ratio of at least 2.0 (HER2/CEP17 ratio $\geq 2.0$ ). Because of the rarity of concomitant HER 2 mutation and amplification in lung adenocarcinoma, only a few studies are currently available and clinical selection of the treatment program has been difficult. We presented this case to provide evidence and basis for the clinical therapy of such patients.

\section{Case presentation}

A 64-year-old, previously healthy Chinese woman who had never smoked visited our lung cancer clinic on February 2018 because of a productive cough for 1 week and severe dull pain in the lumbar area for 2 days. Computed tomography (CT) of the chest showed a nodular highdensity shadow (about $4.2 \times 2.6 \mathrm{~cm}$ in size) in the right lower lobe, and unclear pleural effusion (Figure 1). Multiple cuts of abdominal CT showed bilateral adrenal metastases. Finally, she was diagnosed with stage IV lung cancer by CT-guided percutaneous lung puncture; specifically, the pathologic examination demonstrated adenocarcinoma with an acinar and micropapillary pattern (Figure 2).

Comprehensive genomic profiling of the percutaneous lung puncture specimens was performed using ARMS. The results showed the presence of $H E R 2$ gene mutation, but there were no mutations in the EGFR, KRAS, BRAF, NRAS, and PIK3CA genes and no rearrangements in the anaplastic lymphoma kinase (ALK), ROS1, and RET genes. Thereafter, Sanger sequencing identified insertion mutations in exon 20 of the HER 2 gene (c.2326G > TTGT) (Figure 3). The presence of HER 2 gene amplification was defined by FISH, which showed a HER2/CEP17 ratio of 2.6 in a total of 40 lung cancer cells (Figure 4).

The patient decided against chemotherapy and agreed to 


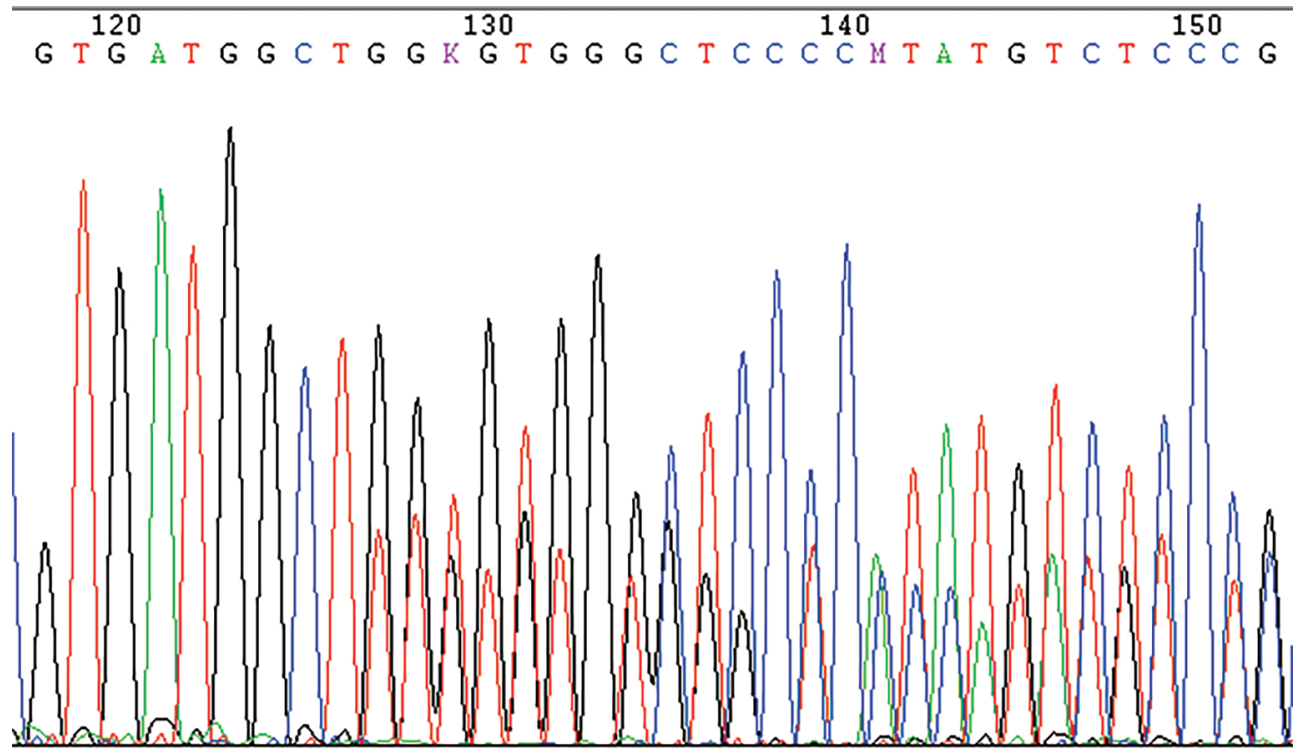

Figure 3 Sanger sequencing results. Mutations are inserted in exon 20 of the HER2 gene (c.2326G > TTGT). HER2, human epidermal growth factor receptor 2 .

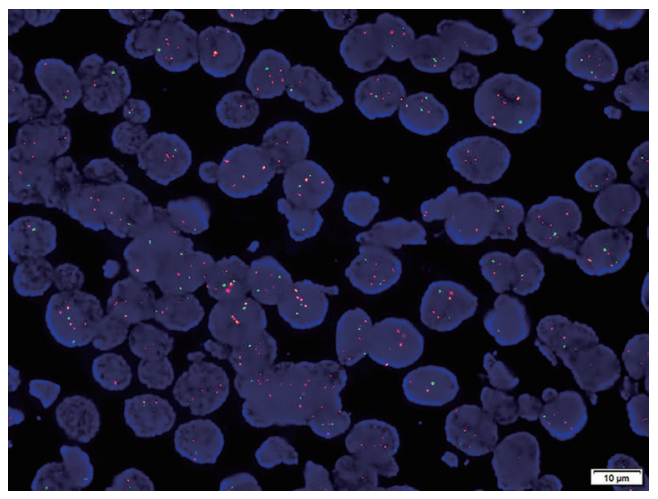

Figure 4 FISH results. HER2 gene amplification (HER2/CEP17 ratio of 2.6). FISH, fluorescence in situ hybridization; HER2, human epidermal growth factor receptor 2 .

receive targeted therapy. On the basis of the findings that the patient harbored HER2 mutation and amplification, oral afatinib was started at a dose of $40 \mathrm{mg}$ daily as firstline therapy from March 2018. After 2 months, the tumor decreased in size (Figure 1) and Clinical symptoms were also relieved. The patient was followed up at outpatient clinic every 2 months and achieved partial response (PR) in response to afatinib until October 2018, she complained of headaches, and her family noticed changes in personality. Brain magnetic resonance imaging showed multiple brain metastases. However, there was no obvious change in the right lung lesions (Figure 1). She continued to receive afatinib combined with brain radiotherapy. Unfortunately, the patient subsequently died of brain failure in January 2019 (The timeline see Figure 5).

\section{Discussion}

We presented an unusual case of a female patient with lung adenocarcinoma harboring HER 2 mutation and amplification simultaneously. To the best of our knowledge, there have been no reported cases of targeted therapy for lung adenocarcinoma with coexisting HER2 mutation and amplification. With the advent of targeted therapy, EGFRtyrosine kinase inhibitors (TKIs) and ALK inhibitors have become the first-line treatment for advanced NSCLC, with significant improvements in overall survival and progression-free survival (8). Personalized targeted therapy is based on the mutation types, with different mutations requiring different treatment. Our report showed afatinib has a relatively good effect on the pulmonary lesion of this case, the patient achieved PR after afatinib treatment. Therefore, after the occurrence of brain metastasis, afatinib is still used on the basis of brain radiotherapy, instead of other targeted drugs. However, afatinib does not appear to be effective against brain metastases from lung cancer, due to the patients eventually died of brain failure.

Our review of a considerable amount of available 


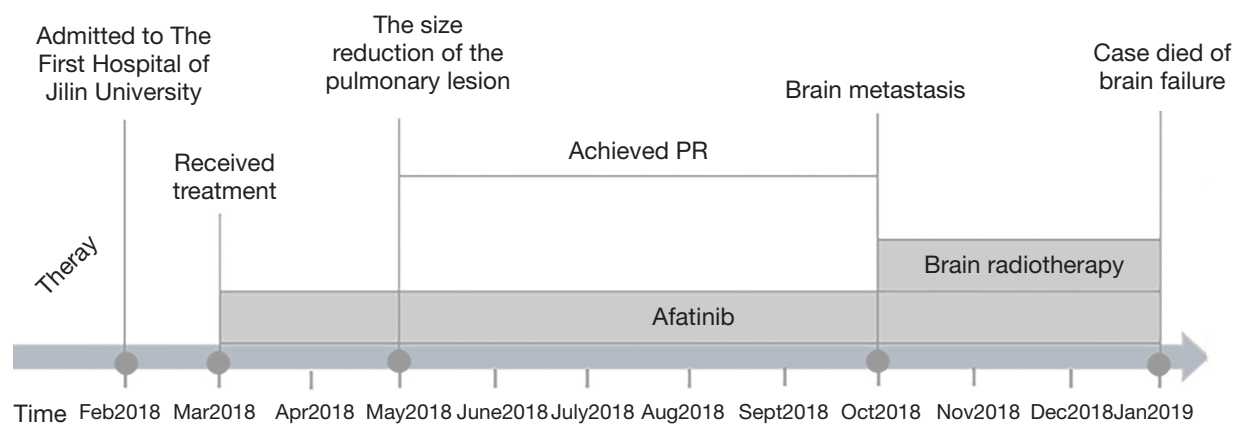

Figure 5 Timeline. The information of the patient with advanced lung adenocarcinoma. PR, partial response.

literature indicated that targeted therapy for the HER2 gene of NSCLC remains under investigation, and the optimal treatment for specific HER2 targets has not been clinically determined (9). The National Comprehensive Cancer Network has described the anti-HER2 antibody trastuzumab and the EGFR-TKI afatinib as potential candidates for the treatment of HER2-mutant NSCLC. In addition, Mazières et al. (10) demonstrated that trastuzumab and afatinib had certain effects on NSCLC with insertion mutation in the HER2 gene exon 20; specifically, the objective response rates (ORRs) to trastuzumab and afatinib were $57 \%$ and $33 \%$, respectively, but there was no response to lapatinib or masitinib.

Afatinib is a potent blocker of the ERBB family and covalently binds to the kinase domains of EGFR, HER2, and HER4, resulting in irreversible inhibition of tyrosine kinase autophosphorylation. In a recent phase II clinical trial (11), afatinib was reported to have resulted in a $71 \%$ disease control rate (DCR) in patients with HER2 mutation and a certain effect in patients with EGFR amplification. In a study by De Grève et al. (12), all three patients who received targeted therapy with afatinib showed good ORRs, and all had exon 20 insertion mutations. For HER2 amplification in patients with NSCLC, Ross et al. (13) found that afatinib did not have a definitive effect. Trastuzumab is an anti-HER2 monoclonal antibody that is currently used in breast cancer patients with HER 2 amplification and overexpression, but its use in HER2-positive NSCLC is not well defined. Cappuzzo et al. (14) reported that trastuzumab was effective for exon 20 insertion mutations. The European EUHER2 cohort study (15) showed that the ORR and DCR values for 57 patients with HER2 mutation were $50.9 \%$ and $79.5 \%$, respectively. In a randomized phase II trial (16), trastuzumab chemotherapy was well tolerated by patients with HER2-positive NSCLC, but no clinical efficacy was observed. At present, there is an ongoing study on trastuzumab for the treatment of HER 2 amplification.

In this case, the patient was identified as having HER2 gene mutation combined with amplification. In view of the rarity of such a case and the more expensive price of trastuzumab, compared with that of afatinib, the patient received afatinib as a trial therapy. Follow-up showed stability of the lung cancer lesions during treatment with afatinib. Unfortunately, brain metastasis developed later on and subsequently led to brain failure and death; this may have been related to the inability of afatinib to penetrate the blood-brain barrier and control the brain lesions $(17,18)$. Because trastuzumab also failed to penetrate the bloodbrain barrier and afatinib controlled the stability of the lung lesions, treatment with trastuzumab was not considered.

The management of the case may serve as a reference for clinicians to select the optimal agent. In this case the efficacy of afatinib in controlling lung lesions can be observed. Therefore, afatinib could be a potential treatment option for subgroups of advanced lung adenocarcinoma with coexisting HER2 mutation and amplification.

\section{Acknowledgments}

Funding: None.

\section{Footnote}

Conflicts of Interest: The authors have no conflicts of interest to declare.

Ethical Statement: The authors are accountable for all aspects of the work in ensuring that questions related to the accuracy or integrity of any part of the work are appropriately investigated and resolved. Written informed 
consent was obtained from the patient for publication of this manuscript and any accompanying images.

Open Access Statement: This is an Open Access article distributed in accordance with the Creative Commons Attribution-NonCommercial-NoDerivs 4.0 International License (CC BY-NC-ND 4.0), which permits the noncommercial replication and distribution of the article with the strict proviso that no changes or edits are made and the original work is properly cited (including links to both the formal publication through the relevant DOI and the license). See: https://creativecommons.org/licenses/by-nc-nd/4.0/.

\section{References}

1. Thunnissen E, van der Oord K, den Bakker M. Prognostic and predictive biomarkers in lung cancer. A review. Virchows Arch 2014;464:347-58.

2. Wang SE, Narasanna A, Perez-Torres M, et al. HER2 kinase domain mutation results in constitutive phosphorylation and activation of HER2 and EGFR and resistance to EGFR tyrosine kinase inhibitors. Cancer Cell 2006;10:25-38.

3. Notsuda H, Bradbury PA, Tsao MS. HER2 transmembrane domain mutations: rare new target for non-small cell lung cancer therapy. J Thorac Oncol 2017;12:422-4.

4. Mar N, Vredenburgh JJ, Wasser JS. Targeting HER2 in the treatment of non-small cell lung cancer. Lung Cancer 2015;87:220-5.

5. Li BT, Ross DS, Aisner DL, et al. HER2 amplification and HER2 mutation are distinct molecular targets in lung cancers. J Thorac Oncol 2016;11:414-9.

6. Arcila ME, Chaft JE, Nafa K, et al. Prevalence, clinicopathologic associations, and molecular spectrum of ERBB2 (HER2) tyrosine kinase mutations in lung adenocarcinomas. Clin Cancer Res 2012;18:4910-8.

7. Suzuki M, Shiraishi K, Yoshida A, et al. HER2 gene mutations in non-small cell lung carcinomas: concurrence with Her2 gene amplification and Her2 protein expression and phosphorylation. Lung Cancer 2015;87:14-22.

8. Brückl W, Tufman A, Huber RM. Advanced non-small cell lung cancer (NSCLC) with activating EGFR mutations: first-line treatment with afatinib and other EGFR TKIs. Expert Rev Anticancer Ther 2017;17:143-55.

9. Takezawa K, Pirazzoli V, Arcila ME, et al. HER2 amplification: a potential mechanism of acquired resistance to EGFR inhibition in EGFR-mutant lung cancers that lack the second-site EGFRT790M mutation. Cancer Discov 2012;2:922-33.

10. Mazières J, Peters S, Lepage $B$, et al. Lung cancer that harbors an HER2 mutation: epidemiologic characteristics and therapeutic perspectives. J Clin Oncol 2013;31:1997-2003.

11. De Grève J, Moran T, Graas MP, et al. Phase II study of afatinib, an irreversible ErbB family blocker, in demographically and genotypically defined lung adenocarcinoma. Lung Cancer 2015;88:63-9.

12. De Grève J, Teugels E, Geers C, et al. Clinical activity of afatinib (BIBW 2992) in patients with lung adenocarcinoma with mutations in the kinase domain of HER2/neu. Lung Cancer 2012;76:123-7.

13. Ross HJ, Blumenschein GR Jr, Aisner J, et al. Randomized phase II multicenter trial of two schedules of lapatinib as first- or second-line monotherapy in patients with advanced or metastatic non-small cell lung cancer. Clin Cancer Res 2010;16:1938-49.

14. Cappuzzo F, Bemis L, Varella-Garcia M. HER2 mutation and response to trastuzumab therapy in non-small-cell lung cancer. N Engl J Med 2006;354:2619-21.

15. Mazières J, Barlesi F, Filleron T, et al. Lung cancer patients with HER2 mutations treated with chemotherapy and HER2-targeted drugs: results from the European EUHER2 cohort. Ann Oncol 2016;27:281-6.

16. Gatzemeier U, Groth G, Butts C, et al. Randomized phase II trial of gemcitabine-cisplatin with or without trastuzumab in HER2-positive non-small-cell lung cancer. Ann Oncol 2004;15:19-27.

17. Zhou L, He J, Xiong W, et al. Impact of whole brain radiation therapy on CSF penetration ability of Icotinib in EGFR-mutated non-small cell lung cancer patients with brain metastases: results of phase I dose-escalation study. Lung Cancer 2016;96:93-100.

18. Fang L, Sun X, Song Y, et al. Whole-brain radiation fails to boost intracerebral gefitinib concentration in patients with brain metastatic non-small cell lung cancer: a selfcontrolled, pilot study. Cancer Chemother Pharmacol 2015;76:873-7.

Cite this article as: Liu X, Cao Y, Li Y, Duan X. Advanced lung adenocarcinoma with coexistent HER2 mutation and amplification and response to afatinib: a case report. Ann Palliat Med 2020;9(2):483-487. doi: 10.21037/apm.2020.02.14 